This copy is for your personal, non-commercial use only.

If you wish to distribute this article to others, you can order high-quality copies for your colleagues, clients, or customers by clicking here.

Permission to republish or repurpose articles or portions of articles can be obtained by following the guidelines here.

The following resources related to this article are available online at www.sciencemag.org (this information is current as of May 8, 2012):

Updated information and services, including high-resolution figures, can be found in the online version of this article at:

http://www.sciencemag.org/content/308/5720/416.full.html

Supporting Online Material can be found at:

http://www.sciencemag.org/content/suppl/2005/04/11/308.5720.416.DC1.html

This article has been cited by 49 article(s) on the ISI Web of Science

This article has been cited by 16 articles hosted by HighWire Press; see:

http://www.sciencemag.org/content/308/5720/416.full.html\#related-urls

This article appears in the following subject collections:

Neuroscience

http://www.sciencemag.org/cgi/collection/neuroscience 


\title{
Representation of Visual Gravitational Motion in the Human Vestibular Cortex
}

\author{
Iole Indovina, ${ }^{1,2}$ Vincenzo Maffei, ${ }^{1}$ Gianfranco Bosco, ${ }^{3}$ \\ Myrka Zago, ${ }^{1}$ Emiliano Macaluso, ${ }^{2}$ Francesco Lacquaniti ${ }^{1,3,4 *}$
}

\begin{abstract}
How do we perceive the visual motion of objects that are accelerated by gravity? We propose that, because vision is poorly sensitive to accelerations, an internal model that calculates the effects of gravity is derived from graviceptive information, is stored in the vestibular cortex, and is activated by visual motion that appears to be coherent with natural gravity. The acceleration of visual targets was manipulated while brain activity was measured using functional magnetic resonance imaging. In agreement with the internal model hypothesis, we found that the vestibular network was selectively engaged when acceleration was consistent with natural gravity. These findings demonstrate that predictive mechanisms of physical laws of motion are represented in the human brain.
\end{abstract}

The perception of motion is a fundamental property of the visual system. One of the most frequently encountered stimuli is an object accelerated by gravity, as in free-fall, ballistic, pendulum, or wave motion. Survival in the forest, like success on the sports field, critically depends on the ability to estimate the time to collision $[\mathrm{TTC}(t)]$ for accelerated objects, and to react quickly and appropriately as a result. However, psychophysics shows that the human visual system poorly estimates arbitrary accelerations of targets (1-3), and these accelerations generally are not taken into account in timing manual interceptions (4). Moreover, electrophysiological recordings in the monkey show that neurons in a key visual motion area (MT) accurately encode target direction and speed, but they contain only partial information about acceleration (5). Yet, visually guided interceptions of objects falling under gravity are accurately timed (6-8), in contrast with interceptions of objects dropped in microgravity (9). Furthermore, visual gravity cues contribute to perception of causality and naturalness of motion $(10,11)$ and to perception of distance and size for falling objects (12) or biological motion (13). Gravity cues also influence the realism of special effects in cinematography (12). The ability to detect gravitational acceleration in visual motion can be demonstrated early in

${ }^{1}$ Department of Neuromotor Physiology, Scientific Institute Foundation Santa Lucia, via Ardeatina 306, 00179 Rome, Italy. ${ }^{2}$ Department of Neuroimaging, Scientific Institute Foundation Santa Lucia, via Ardeatina 306, 00179 Rome, Italy. ${ }^{3}$ Department of Neuroscience, University of Rome Tor Vergata, Via Montpellier 1, 00133 Rome, Italy. ${ }^{4}$ Center of Space Biomedicine, University of Rome Tor Vergata, Via O. Raimondo 8, 00173 Rome, Italy.

*To whom correspondence should be addressed. E-mail: lacquaniti@caspur.it life. Between 5 and 7 months, infants begin to implicitly expect a downwardly moving object to accelerate and an upwardly moving object to decelerate (10). Therefore, there is ample evidence that gravitational acceleration is taken into account in visual perception and interceptive responses. However, the neural bases of gravitational visual processing are unknown.

Here we propose that an internal model calculating the effects of gravity ( $1 g$ model) on seen objects is derived from graviceptive information, is stored in the vestibular cortex, and is activated by visual motion that appears to be coherent with natural gravity [Fig. 1A and (14)]. The basis for this hypothesis is that the vestibular system is able to estimate the gravity vector in head coordinates by combining signals from otoliths and semicircular canals (14-16) and that multisensory neurons in vestibular cortex also respond to visual stimuli (17). We surmise that, through experience, the vestibular estimate of Earth's gravity is transformed and stored as an abstract representation of gravity accessible by the visual system. Initial evidence for represented visual gravity was provided by the observation that, in the absence of gravitydetermined sensory cues, astronauts initially expect the effects of Earth's gravity on a dropped object when they attempt to catch it in the Spacelab, and they adapt to the new environment only after a few days of flight (9). To test the internal model hypothesis, we measured motor performance and brain activity during different visual tasks. We predict that when the acceleration of a visual target is coherent with represented natural gravity, the $1 g$ model will enable subjects to compute $\operatorname{TTC}(t)$ accurately, by engaging the vestibular network. Conversely, when target acceleration has the same amplitude as, but opposite direction to, natural gravity, subjects will compute TTC $(t)$ less accurately, relying on visual motion areas that are poorly sensitive to arbitrary accelerations.

A first functional magnetic resonance imaging (fMRI) experiment involved three different tasks with a block design. In all tasks, subjects were presented with the picture of a woman standing in front of a building and were asked to maintain fixation on a dot placed just above the woman's head (18) (Movie S1). A ball moved upward from the fixation point at a constant acceleration bounced on the building cornice and returned downward. Initial ball speed was randomized to make flight duration unpredictable from trial to trial. Additionally, the fixation point expanded transiently after a random delay from the end of ball motion. The ball underwent the same average speed changes in all trials, but gravity was directed either toward the ground of the picture ( $1 g$ trials, natural gravity) or away from it ( $-1 g$ trials, reversed gravity). No cue was given to identify either trial type, and perceptually overt differences between them were subtle. In different blocks, subjects were asked either to press a button so as to intercept the descending ball at the time of arrival at the fixation point (proactive task), or to press the button as fast as possible after the Go signal corresponding to the expansion of the fixation point (reactive task). By design, response timing was coupled to the law of motion in proactive tasks, but decoupled in reactive tasks (because of the random delay intervening between the end of ball motion and the Go signal). We used these two tasks to verify that differences in brain activity between $1 g$ and $-1 g$ trials would not merely reflect the nature of the motor task or motor errors (19). Finally, in a baseline condition, subjects simply fixated the expansions of the fixation point, and there was no ball motion in the visual display (No-motion task).

The proactive task required that subjects programmed motor responses before ball landing to compensate for neuromechanical transmission delays (20). Analysis of response times showed that the direction of visual gravity significantly affected the subject's ability to intercept the ball (Fig. 1B, black bars). Thus, all subjects correctly estimated TTC $(t)$ in $1 g$ trials only, and in these trials, the response times were explained by the $1 g$ model that incorporates gravity effects on target motion $(21,22)$. The response times for $-1 g$ trials were explained by the $\tau$ model that incorporates information about target position and velocity, but ignores acceleration $(4,21-23)$. As expected, the direction of gravity had little effect on response times during the reactive task (Fig. 1B, white bars).

Analysis of fMRI data showed that $1 g$ trials were associated with significantly more activity than $-1 g$ trials in a network involv- 
ing the insular cortex, temporoparietal junction, premotor and supplementary motor areas (SMA), middle cingulate cortex, postcentral gyrus, posterior thalamus, putamen, and medial cerebellum (main effect of $1 g$ motion, Fig. 2, tables S1 and S2). In agreement with the internal model hypothesis, the network processing $1 g$ visual motion included the insulae and temporoparietal junctions, which are generally considered the core regions of the vestibular cortex $[(24,25)$; see also (17) for related work in nonhuman primates]. Activation of the vestibular network was observed during both proactive and reactive tasks, which suggests that it depends on the presence of natural gravity in the visual stimuli, rather than on the specific motor task (anticipatory ball interception versus reaction-time response). Anatomical localization of the vestibular cortex was carried out by means of a second experiment. Standard caloric vestibular stimulations (alternating cold irrigations of the left and right ear) were performed blindfolded, resulting in vestibular sensations and nystagmus (18) that are known to depend on canal-otolith interactions performed by vestibular internal models (26). The resulting pattern of brain activation (tables S1 and S2) closely matched that reported in several previous neuroimaging studies involving caloric or galvanic vestibular stimulations $(18,24,27)$. We statistically assessed what brain regions were activated by both $1 g$ visual motion and vestibular stimulation (Fig. 2, A and B; table $\mathrm{S} 1$ ). This analysis revealed a common network composed of insular cortex (posterior insula and retroinsula), temporoparietal junction, ventral premotor area, SMA, middle cingulate cortex, and postcentral gyrus, as well as posterior thalamus and putamen. Our findings demonstrate that the vestibular network is involved in processing visual motion when this is coherent with natural gravity, which supports the hypothesis of an internal $1 g$ model.

As for target motion unrelated to natural gravity, our hypothesis predicts that there should be less involvement of the internal $1 g$ model and, hence, less activation of the vestibular network. Indeed, fMRI analysis showed that $-1 g$ trials were associated with significantly greater activity than $1 g$ trials in a region located around the lateral occipital sulcus (LOS) in the middle and inferior occipital gyri (fig. S1, table S3), a region previously identified as motion-sensitive $(28,29)$. In the context of our tasks, LOS may help compute TTC $(t)$ from target position and velocity while ignoring acceleration, as implied by the $\tau$ model $(4,23,30)$. As expected, vestibular stimulation did not activate this area, which emphasizes the segregation between neural representations of natural visual gravity and those of visual motion unrelated to gravity. Note that in contrast with gravity information, low-level motion cues were comparable between $1 g$ and $-1 g$ trials, because the visual target moved through the same path and with the same average speed changes in both trial types. Accordingly, we found that several visual motion cortical areas were activated by both $1 g$ and $-1 g$ trials when these trials were compared with the baseline (No-motion) condition. Visual motion areas included occipital area hV3a, middle temporal area $\mathrm{hMT} / \mathrm{V} 5+$, and intraparietal sulcus regions $(28,29)$. Moreover, several other areas related to the sensorimotor aspects of the tasks were also activated in this comparison (including superior parietal as well as frontal premotor and motor areas).

In summary, we showed that brain regions overlapping with those activated by direct vestibular stimuli are activated when the internal $1 g$ model is called into play by visual exomotion coherent with natural gravity, even if there is no time-varying stimulation of the vestibular sensors. In monkeys, the parietoinsular vestibular cortex (PIVC) at the posterior end of the insula is the core region of the vestibular cortex, as it contains the greatest number of vestibular-driven neurons $(17,31)$.

Most PIVC neurons respond jointly to head accelerations and to optokinetic and neck somatosensory stimuli. PIVC is reciprocally connected (via ventroposterior thalamus) with the vestibular nuclei, as well as with the other vestibular cortical regions [in temporoparietal junction, postcentral gyrus, and ventral premotor and cingulate cortices (17)]. In addition, PIVC receives anatomical projections from the pulvinar, inferior parietal, and superior temporal areas, and this provides possible routes for visual inputs to vestibular cortex, as implied by the visual responses reported here (Fig. 2, A and B). In humans, lesions of posterior insula and retroinsula (probable homologs of monkey PIVC) lead to a tilt of the perceived visual vertical and rotational vertigo (25). Focal electrical stimulation elicits sensations of altered gravity or body tilt (32). Accordingly, it has been suggested that the cortical vestibular network is involved in the perception of our spatial orientation relative to the gravitational vertical $(17,24,25,27)$.

Here we propose a new function for this network, namely, a representation of the phys-
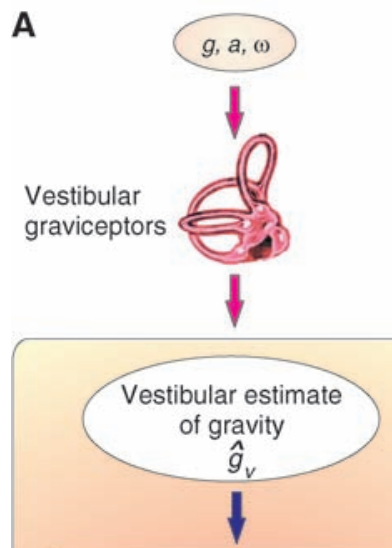

Change of reference frame
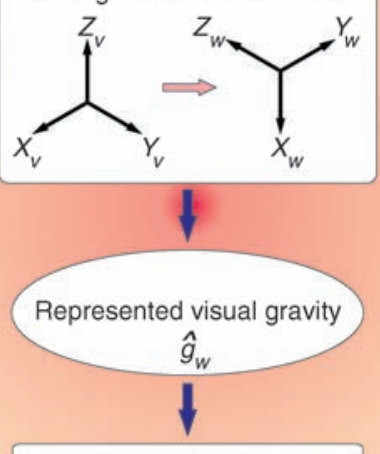

Internalized

Newton's laws

Internal $1 \mathrm{~g}$ model in the vestibular network
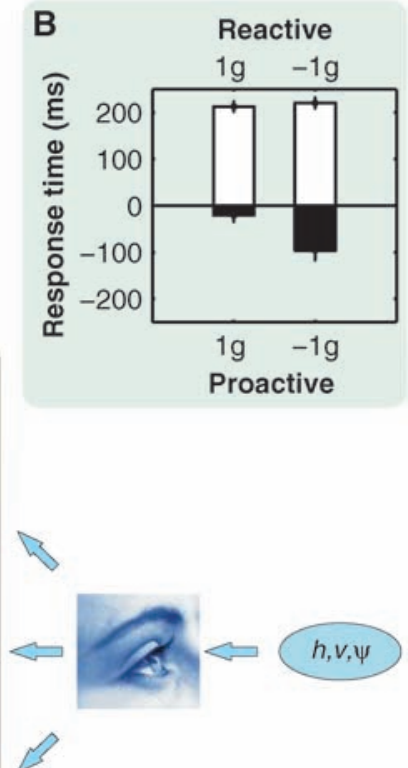

Fig. 1. (A) Neural computations predicted by the hypothesis of the internal model of gravity. The vestibular semicircular canals measure the angular velocity of the head $(\omega)$; the otolith organs measure both gravity $(g)$ and linear acceleration of the head (a). Internal model calculations are included within the yellow and pink box. A vestibular estimate of gravity $\left(\hat{g}_{v}\right)$ is computed in headfixed coordinates $\left(X_{v}\right.$, $\left.Y_{v}, Z_{v}\right)$ by the CNS. In general, rotational optokinetic cues $(\psi)$ and extravestibular graviceptive cues may also contribute toward computing $\hat{g}_{v}$. An abstract representation of gravity $\left(\hat{g}_{w}\right)$ accessible by the visual system is constructed by a change of reference frame to world-fixed coordinates $\left(X_{w}, Y_{w}\right.$ $Z_{w}$ ), so that it matches the perceived top-bottom axis $\left(Z_{w}\right)$ of the visual scene. The internal model of Newton's laws results from the combination of $\hat{g}_{w}$ with on-line visual estimates about target motion ( $h$ and $v$ are the height of the target above the interception point and target velocity, respectively) and can be used by the brain for different scopes, such as predicting target $\operatorname{TTC}(t)$, or perceiving a motion as natural. For more details, see (14). (B) Response times (RT) are means ( \pm SEM, $n=17)$ for proactive (black bars) and reactive (white bars) tasks. Negative time values in proactive correspond to responses occurring before the arrival time of the ball; positive time values in reactive correspond to responses after the Go signal. RT was significantly $(P<0.001)$ different from 0 in $-1 g$ proactive, $1 g$ reactive, and $-1 g$ reactive trials. 


\section{R E P O R T S} sorimotor coordination might share an internal $1 g$ model permanently stored in the distributed vestibular network. In this network, sensory processing may not be determined by modality but by the nature of the input, i.e., gravitational acceleration. Thus, the vestibular estimate of gravity helps in discriminating tilt from translation of the head $(15,16)$, and the $1 g$ model removes ambiguity from visual information in TTC estimates (as shown ical laws of motion. Different forms of sen-

here). Furthermore, the areas of somatosensory cortex and ventral premotor cortex activated by visual $1 g$ motion overlap with sensory and motor arm and hand representations, which suggests that the internal $1 g$ model is also used to account for gravitational effects on arm position when planning arm movements or sensing arm position (kinesthesia), in agreement with the notion of multimodal processing in the cortical vestibular system $(17,24,27)$. Finally, the

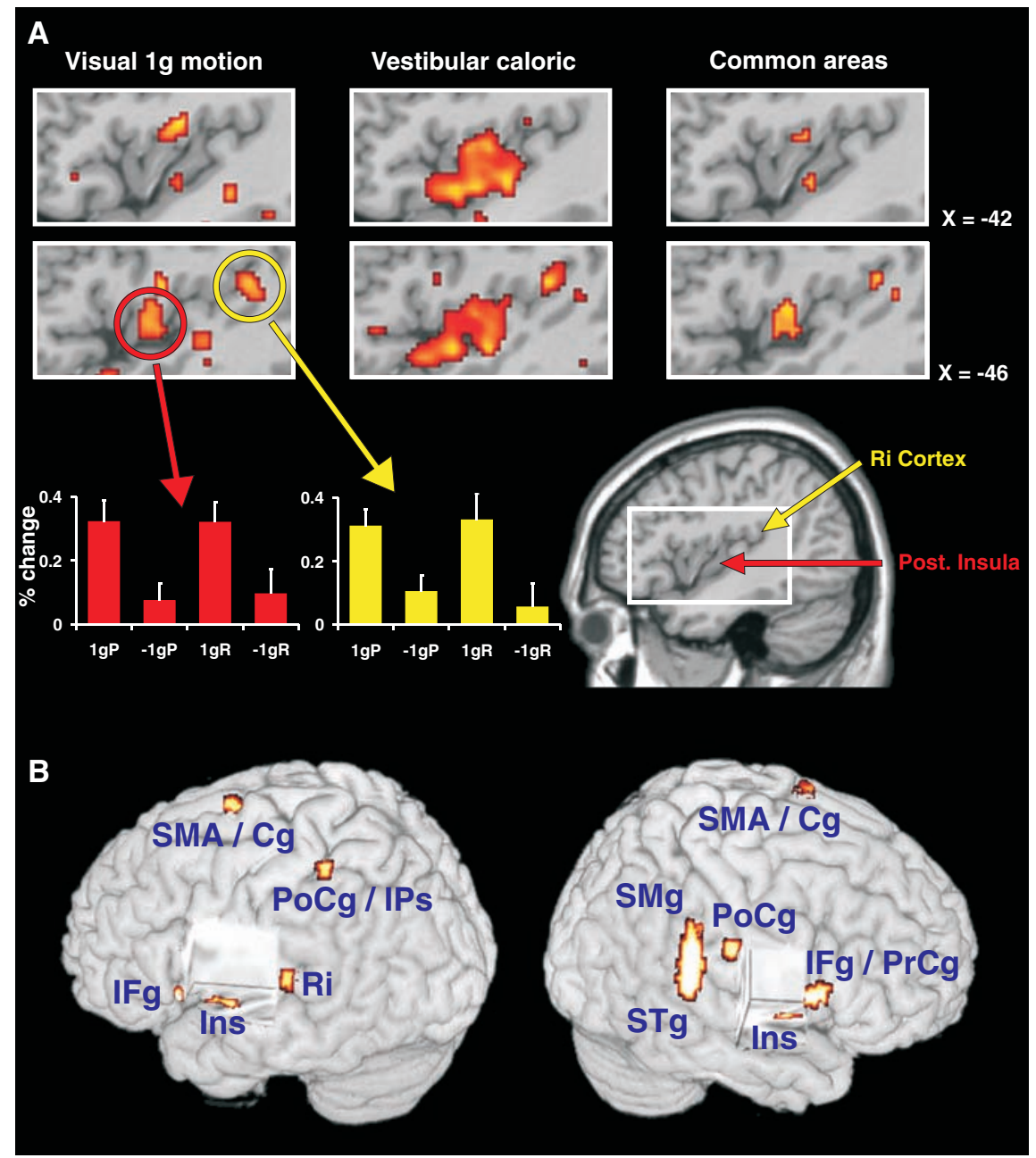

Fig. 2. (A) Common areas observed in the insulae for $1 \mathrm{~g}$ visual-motion stimuli and direct vestibular stimuli. (Top) Color-coded areas of activation ( $P<0.05$, corrected for multiple comparisons) at two different parasagittal sections in the left hemisphere. From left to right: main effect of $1 \mathrm{~g}$ motion $\{[(1 g P)+(1 g R)]>[(-1 g P)+(-1 g R)]\}$, main effect of caloric vestibular stimulation [(left stimuli + right stimuli) > baseline], and common activation for visual $1 \mathrm{~g}$ motion and caloric vestibular stimulation. (Bottom) (right) The rectangle delimits the areas depicted in the above panels. Red and yellow arrows point to the anatomical location of the posterior insula and retroinsular cortex on a sagittal section $(x=-42 \mathrm{~mm})$ of the Montreal Neurological Institute (MNI) template in stereotactic space. (Bottom) (left) Mean activity $( \pm$ SEM, $n=17)$ for the left insula cluster (95 voxels centered around the central insular sulcus) and the left retroinsular cluster (196 voxels at the temporoparietal junction). For each active task, averaged activity within these clusters is expressed as activation compared to baseline (No motion), in percentage, with respect to whole-brain mean. For coordinates of peak activations, see table $\mathrm{S} 1.1 g \mathrm{P},-1 g \mathrm{P}, 1 g \mathrm{R}$, and $-1 g \mathrm{R}$ denote $1 g$ proactive, $-1 g$ proactive, $1 g$ reactive, and $-1 g$ reactive trials, respectively. (B) Overall cortical network of common activations for visual $1 \mathrm{~g}$ motion and caloric vestibular stimulation, projected onto the surface-rendered $\mathrm{MNI}$ template. A perisylvian volume of brain is removed to show the insular region, deep in the lateral sulcus. $\mathrm{Cg}$, middle cingulate gyrus; IFg, inferior frontal gyrus; Ins, insula; IPs, intraparietal sulcus; PoCg, postcentral gyrus; $\operatorname{PrCg}$, precentral gyrus; Ri, retroinsula; SMA, supplementary motor area; $S M g$, supramarginal gyrus; and STg, superior temporal gyrus. For coordinates of peak activations, see table S1. internal $1 \mathrm{~g}$ model might influence cognitive processes, transforming gravity into an abstract reference within the brain that contributes to our mind's balance (33). The idea that organisms' perception is tuned to environmental constraints by means of their internalization has long been around in psychology (34). Here we provided direct evidence that the fundamental physical constraint of Earth's gravity is internalized in the human brain.

\section{References and Notes}

1. J. T. Todd, J. Exp. Psychol. Hum. Percept. Perform. 7, 975 (1981).

2. P. Werkhoven, H. P. Snippe, A. Toet, Vision Res. 32 , 2313 (1992)

3. A. M. Brouwer, E. Brenner, J. B. Smeets, Percept. Psychophys. 64, 1160 (2002)

4. N. L. Port, D. Lee, P. Dassonville, A. P. Georgopoulos, Exp. Brain Res. 116, 406 (1997).

5. S. G. Lisberger, J. A. Movshon, J. Neurosci. 19, 2224 (1999).

6. F. Lacquaniti, C. Maioli, J. Neurosci. 9, 134 (1989).

7. F. Lacquaniti, M. Carrozzo, N. A. Borghese, in Multisensory Control of Movement, A. Berthoz, Ed. (Oxford Univ. Press, Oxford, 1993), pp. 379-393.

8. M. K. McBeath, D. M. Shaffer, M. K. Kaiser, Science 268, 569 (1995).

9. J. Mclntyre, M. Zago, A. Berthoz, F. Lacquaniti, Nat. Neurosci. 4, 693 (2001).

10. I. K. Kim, E. S. Spelke, J. Exp. Psychol. Hum. Percept. Perform. 18, 385 (1992).

11. C. R. Twardy, G. P. Bingham, Percept. Psychophys. 64 956 (2002).

12. J. S. Watson, M. S. Banks, C. von Hofsten, C. S. Royden, Perception 21, 69 (1992).

13. D. Jokisch, N. F. Troje, J. Vis. 3, 252 (2003).

14. The vestibular semicircular canals measure the angular velocity of the head $(\omega)$; the otolith organs measure gravitoinertial acceleration $(f=g-a)$. A vestibular estimate of gravity $\left(\hat{g}_{v}\right)$ is computed by the central nervous system (CNS) as a Bayesian weighted average of multicue information: $\hat{g}_{v}=\sum_{i} \tilde{g}_{i}$ where $\tilde{g}_{i}$ is the estimate of $g$ from the $i$ th sensor (35). In general, $\hat{g}_{v}$ can be obtained from (i) the neural solution of the nonlinear integral $\int\left(\hat{g}_{v} \times \hat{\omega}_{v}\right) d t$ provided by an internal model of how $\omega$ and $g$ interact physically ( $\times$ designates the vector cross product), (ii) the internal model discrimination between gravity and linear acceleration $(g=f+a)$, (iii) the idiotropic vector aligned with the rostrocaudal body axis, (iv) rotational optokinetic cues $(\psi)$, and $(v)$ somatosensory cues. An abstract representation of gravity $\left(\hat{g}_{w}\right)$ accessible by the visual system is constructed by a change of reference frame from head-fixed $\left(X_{v}, Y_{v}, Z_{v}\right)$ to world-fixed $\left(X_{w}, Y_{w}, Z_{w}\right)$ coordinates. The internal model of Newton's laws results from the combination of $\hat{g}_{w}$ with on-line visual estimates about target motion.

$$
\operatorname{TTC}(t)=\frac{-\hat{v}_{w}(t)+\sqrt{\hat{v}_{w}(t)^{2}+2 \hat{g}_{w} \hat{h}_{w}(t)}}{\hat{g}_{w}}
$$

where $\operatorname{TTC}(t)$ is the estimate of the remaining time-to-arrival of the target at the interception point at any time $t$ during descent, $\hat{h}_{w}(t)$ and $\hat{v}_{w}(t)$ are the visually estimated height of the target above the interception point and target velocity, respectively. Note that $\hat{h}_{w}(t)$ and $\hat{v}_{w}(t)$ in Eq. 1 must be in the same reference frame and on the same scale as $\hat{g}_{w}$. Thus, either all variables are defined in world coordinates by scaling retinal coordinates of the target by perceived extent (or apparent distance) of target trajectory, or all variables are defined in retinal coordinates, and $\hat{g}_{w}$ must be scaled accordingly. The computations outlined above do not all occur necessarily on-line. In the present experiments, subjects lay supine, $a=\omega=$ $\psi=0$, and $\hat{g}_{w}$ was roughly orthogonal to $g$.

15. D. M. Merfeld, L. Zupan, R. J. Peterka, Nature 398 615 (1999). 
16. D. E. Angelaki, A. G. Shaikh, A. M. Green, J. D. Dickman, Nature 430, 560 (2004).

17. W. O. Guldin, O. J. Grusser, Trends Neurosci. 21, 254 (1998).

18. Materials and methods are available as supporting material on Science Online.

19. H. Imamizu et al., Nature 403, 192 (2000).

20. A mean visuomotor processing time of $171 \mathrm{~ms}$ was estimated for both $1 g$ and $-1 g$ trials, on the basis of methods described in $(4,22)$.

21 . Both the $1 \mathrm{~g}$ model and the $\tau$ model use on-line visual information about target position and velocity to trigger an interceptive movement when TTC $(t)$ [see (14)] reaches a time threshold equal to the visuomotor processing time (22). However, the $1 \mathrm{~g}$ model computes $\operatorname{TTC}(t)$ under the assumption that the target is accelerated by gravity [Eq. 1 in (14)], whereas the $\tau$ model assumes that the target moves at constant speed $\left[\operatorname{TTC}(t)=h_{w}(t) / \hat{v}_{w}(t)\right.$, see $\left.(4,23)\right]$. Therefore, if the brain uses the $1 \mathrm{~g}$ model to predict target motion, the responses to $1 g$ trials will be correctly timed. By contrast, the $\tau$ model overestimates the TTC $(t)$ of $1 g$ trials and predicts that the corresponding responses should be systematically late. As a quantitative test, the time of response was computed separately for the five flight durations and was averaged across all trials of all subjects for a given flight duration. For $1 \mathrm{~g}$ trials, the response times predicted by the $1 \mathrm{~g}$ model were highly correlated with the experimental values in all subjects (mean $r=0.998$ ), whereas those predicted by the $\tau$ model systematically failed to fit the experimental values (mean $r=-0.5$ ). The root mean square error (RMSE) of the fit to $1 g$ trials was worse by a factor of 1.7 for the $\tau$ model than for the $1 g$ model. For $-1 g$ trials, the response times predicted by the $\tau$ model were highly correlated with the experimental values in all subjects (mean $r=0.973$ ), and adequacy of the fit was demonstrated by the random distribution of the residuals around the mean over the data range. Instead, the $1 g$ model fitted the responses to $-1 g$ trials less adequately, as shown by a systematic bias of the residuals around the mean (thus $r$ value was not computed for this fit). Accordingly, the RMSE of the fit was 3.6 times worse for the $1 g$ model than for the $\tau$ model.

22. M. Zago et al., J. Neurophysiol. 91, 1620 (2004)

23. D. N. Lee, P. E. Reddish, Nature 293, 293 (1981).

24. G. Bottini et al., Exp. Brain Res. 99, 164 (1994).

25. T. Brandt, M. Dieterich, A. Danek, Ann. Neurol. 35, 403 (1994).

26. R. J. Peterka, C. C. Gianna-Poulin, L. H. Zupan, D. M. Merfeld, J. Neurophysiol. 92, 2333 (2004).

27. S. Bense, T. Stephan, T. A. Yousry, T. Brandt, M. Dieterich, J. Neurophysiol. 85, 886 (2001).

28. G. A. Orban et al., Neuropsychologia 41, 1757 (2003)

29. G. Rees, K. Friston, C. Koch, Nat. Neurosci. 3, 716 (2000).

30. H. Merchant, A. Battaglia-Mayer, A. P. Georgopoulos, Cereb. Cortex 14, 314 (2004).

31. O. J. Grusser, M. Pause, U. Schreiter, J. Physiol. 430, 537 (1990).

32. O. Blanke, S. Ortigue, T. Landis, M. Seeck, Nature 419, 269 (2002)

33. V. Smetacek, Nature 415, 481 (2002).

34. R. N. Shepard, Psychol. Rev. 91, 417 (1984).

35. L. H. Zupan, D. M. Merfeld, C. Darlot, Biol. Cybern. 86, 209 (2002).

36. We thank R. Frackowiak, C. D. Frith, G. Galati, C. Porro, G. Rizzolatti, and J. N. Sanes for comments and advice on previous versions of this work. Supported by the Italian Ministry of Health, the Italian Ministry of University and Research (FIRB and PRIN grants), and the Italian Space Agency.

Supporting Online Material

www.sciencemag.org/cgi/content/full/308/5720/416/ DC1

Materials and Methods

SOM Text

Fig. S1

Tables S1 to S5

References and Notes

Movie S1

29 November 2004; accepted 31 January 2005 10.1126/science. 1107961

\title{
Complement Factor H Variant Increases the Risk of Age-Related Macular Degeneration
}

\author{
Jonathan L. Haines, ${ }^{1}$ Michael A. Hauser, ${ }^{2}$ Silke Schmidt, ${ }^{2}$ \\ William K. Scott, ${ }^{2}$ Lana M. Olson, ${ }^{1}$ Paul Gallins, ${ }^{2}$ Kylee L. Spencer, ${ }^{1}$ \\ Shu Ying Kwan, ${ }^{2}$ Maher Noureddine, ${ }^{2}$ John R. Gilbert, ${ }^{2}$ \\ Nathalie Schnetz-Boutaud, ${ }^{1}$ Anita Agarwal, ${ }^{3}$ Eric A. Postel, ${ }^{4}$ \\ Margaret A. Pericak-Vance ${ }^{2 *}$
}

\begin{abstract}
Age-related macular degeneration (AMD) is a leading cause of visual impairment and blindness in the elderly whose etiology remains largely unknown. Previous studies identified chromosome 1q32 as harboring a susceptibility locus for AMD. We used single-nucleotide polymorphisms to interrogate this region and identified a strongly associated haplotype in two independent data sets. DNA resequencing of the complement factor $\mathrm{H}$ gene within this haplotype revealed a common coding variant, $\mathrm{Y} 402 \mathrm{H}$, that significantly increases the risk for AMD with odds ratios between 2.45 and 5.57. This common variant likely explains $\sim 43 \%$ of AMD in older adults.
\end{abstract}

AMD causes progressive impairment of central vision and is the leading cause of irreversible vision loss in older Americans (1). The most severe form of AMD involves neovascular/exudative (wet) and/or atrophic (dry) changes to the macula. Although the etiology of AMD remains largely unknown,

${ }^{1}$ Center for Human Genetics Research, Vanderbilt University Medical Center, Nashville, TN 37232, USA. ${ }^{2}$ Center for Human Genetics and Department of Medicine, Duke University Medical Center, DUMC Box 3445, 595 LaSalle Street, Durham, NC 27710 USA. ${ }^{3}$ Vanderbilt Eye Institute, Vanderbilt University Medical Center, Nashville, TN 37232, USA. ${ }^{4}$ Duke University Eye Center and Department of Ophthalmology, Duke University Medical Center, Durham, NC 27710, USA.

*To whom correspondence should be addressed. E-mail: mpv@chg.duhs.duke.edu

Table 1. CFH sequence variants identified in neovascular AMD cases and normal controls. All individuals were homozygous for the AMD-associated GAGGT haplotype. The 24 affected individuals selected for sequencing had severe neovascular disease (grade 5) (12) with diagnosis before age 74 (mean age at diagnosis: 65.8 years). The 24 control individuals selected for sequencing had no evidence of AMD (grade 1) with age at exam after age 64 (mean age at exam: 69.8 years). The six previously identified SNPs are labeled using standard nomenclature. The five previously unknown variants are labeled given their base pair location on chromosome 1, Ensembl build 35. Five SNPs create nonsynonymous amino acid changes within $\mathrm{CFH}$, and five SNPs create synonymous changes. Exon 1 is not translated. n/a, not applicable.

\begin{tabular}{|c|c|c|c|c|}
\hline \multirow{2}{*}{ Location } & \multirow{2}{*}{ SNP ID } & \multirow{2}{*}{ Effect } & \multicolumn{2}{|c|}{ Minor allele frequency (\%) } \\
\hline & & & AMD & Controls \\
\hline Exon 1 & rs3753394 & $\mathrm{n} / \mathrm{a}$ & 18 & 24 \\
\hline Exon 2 & rs800292 & V62I & 0 & 6 \\
\hline Exon 6 & $193,380,486 \mathrm{~A} / \mathrm{G}$ & R232R & 0 & 2 \\
\hline Exon 7 & rs1061147 & A307A & 10 & 38 \\
\hline Exon 8 & $193,390,164 \mathrm{C} / \mathrm{T}$ & H332Y & 0 & 5 \\
\hline Exon 9 & rs1061170 & $\mathrm{Y} 402 \mathrm{H}$ & 94 & 46 \\
\hline Exon 11 & $193,414,604 \mathrm{~A} / \mathrm{G}$ & A473A & 0 & 31 \\
\hline Exon 12 & $193,416,415 \mathrm{~A} / \mathrm{G}$ & T519A & 0 & 2 \\
\hline Exon 14 & rs3753396 & Q672Q & 0 & 23 \\
\hline Exon 18 & $193,438,299 \mathrm{C} / \mathrm{T}$ & $\mathrm{H} 878 \mathrm{H}$ & 6 & 2 \\
\hline Exon 19 & HGVbase 000779895 & E936D & 0 & 23 \\
\hline
\end{tabular}

implicated risk factors include age, ethnicity, smoking, hypertension, obesity, and diet (2). Familial aggregation (3), twin studies (4), segregation analysis (5) suggest that there the disease. The candidate gene approach, vant candidates, has implicated variants in the $A B C A 4, F B L N 6$, and $A P O E$ genes as risk factors for AMD. Replication of the $A B C A 4$ and FBLN6 findings has been difficult, and in to the variants explain a small proporapproach uses a combination of genetic linkage and association to identify previously unknown genes involved in AMD. We participated in a recent collaborative genomewide linkage screen (9) in which chromosome 1q32 which focuses on testing biologically rele- 